\title{
Vulnerabilidade ao Estresse e Estratégias de Enfrentamento: um Estudo Comparativo no Ambiente Hospitalar
}

\author{
Vulnerability to Stress and Coping Strategies: a Comparative Study in the Hospital \\ Environment
}

\section{Vulnerabilidad al Estrés y Estrategias de Afrontamiento: un Estudio Comparativo en el Ambiente Hospitalario}

\author{
Rafael Lima de Matos \\ Marley Rosana Melo de Araújo ${ }^{1}$ \\ Universidade Federal de Sergipe (UFS)
}

\begin{abstract}
Resumo
O trabalho no ambiente hospitalar torna os profissionais de saúde suscetíveis ao estresse ocupacional. Objetivou-se, neste estudo, comparar a vulnerabilidade ao estresse no trabalho e as estratégias de enfrentamento utilizadas por profissionais de saúde de um hospital universitário. Utilizou-se a Escala de Vulnerabilidade ao Estresse (EVENT), a Escala Coping Ocupacional (ECO) e um Questionário Sociodemográfico e Ocupacional. A coleta deu-se por conveniência, de forma anônima, individual e coletivamente, nas dependências do hospital. Participaram 80 profissionais de saúde, subdivididos igualmente em dois grupos, empregados públicos e residentes multiprofissionais. Ambos os grupos apresentaram alta vulnerabilidade ao estresse, e as estratégias de enfrentamento mais utilizadas dirigiam-se à reavaliação cognitiva das situações estressoras, não sendo encontradas diferenças significativas intergrupo. Contudo verificou-se que profissionais mais jovens usam mais recorrentemente a fuga ou evitação de situações estressoras como estratégia de enfrentamento. Apesar do diferencial deste trabalho ter sido investigar residentes, recomendam-se estudos adicionais com este público pouco conhecido.
\end{abstract}

Palavras-chave: profissionais da saúde, vulnerabilidade ao estresse, estratégias de enfrentamento

\begin{abstract}
Working in a hospital environment makes health professionals susceptible to occupational stress. This study aimed to compare vulnerability to stress at work and coping strategies used by health professionals at a university hospital. The Stress Vulnerability Scale (SVS), the Occupational Coping Scale (OCS), and a Demographic and Socio-Occupational Questionnaire were applied. The collection took place at the hospital premises, anonymously, individually, and collectively for convenience. Eighty health professionals joined in, equally divided into two groups, public employees and multiprofessional residents. Both groups showed a high vulnerability to stress and the most used coping strategies aimed at the cognitive reassessment of stressful situations with no significant intergroup differences. However, it was found that younger professionals use escaping or avoidance of stressful situations more frequently as a coping strategy. Although the differential of this work was to investigate residents, additional studies are recommended with this little-known public.

Keywords: health professionals, vulnerability to stress, coping strategies
\end{abstract}

\section{Resumen}

El trabajo en el ambiente hospitalario deja a los profesionales de la salud susceptibles al estrés ocupacional. El objetivo de este estudio fue comparar la vulnerabilidad al estrés en el trabajo y las estrategias de afrontamiento utilizadas por profesionales de la salud de un hospital universitario. Se utilizó la escala de Vulnerabilidad al estrés (EVENT), la Escala Coping Ocupacional (ECO) y un Cuestionario Sociodemográfico y Ocupacional. La colecta fue por conveniencia, de forma anónima, individual y colectivamente, en las dependencias del hospital. Participaron 80 profesionales de la salud, subdivididos igualmente en dos grupos, empleados públicos y residentes multiprofesionales. Ambos los grupos presentaron alta vulnerabilidad al estrés, y las estrategias de afrontamiento más utilizadas se dirigían a la reevaluación cognitiva de las situaciones estresantes, sin encontrar diferencias significativas entre los grupos. Sin embargo, se verificó que los profesionales más jóvenes usan de manera más recurrente la fuga o la evitación de situaciones estresantes como estrategia

\footnotetext{
${ }^{1}$ Endereço para contato: Rua Francisco de Assis D. P. Freitas, 100, apt. 2, Ponto Novo, Aracaju, SE. CEP: 49097-710. E-mail: marleymeloaraujo@gmail.com
} 
de afrontamiento. Aunque el diferencial de este trabajo tenga sido investigar los residentes, se recomiendan estudios adicionales con este grupo poco conocido.

Palabras clave: profesionales de la salud, vulnerabilidad al estrés, estrategias de afrontamiento

\section{Introdução}

O estresse acomete $90 \%$ da população mundial, comprometendo o bem-estar do indivíduo, de modo geral, e contribuindo fortemente para o aparecimento de doenças do apareIho circulatório (Stults-Kolehmainen, 2013), as quais representam uma das principais razões para óbito no Brasil (Mansur \& Favarato, 2011). Por definição, o estresse é um fenômeno psicossocial, relacionado ao funcionamento neurofisiológico, ativado quando ocorre a percepção e avaliação, por parte do indivíduo, de um evento, real ou imaginário, que ameace a sua integridade física e/ou mental (Santos, 2010). Por sua vez, a vulnerabilidade ao estresse diz respeito às situações que tornam os indivíduos suscetíveis ao estresse (Sisto, Baptista, Noronha, \& Santos, 2007), podendo ser definida como distorções cognitivas, caracterizadas por um equívoco, por parte do indivíduo, em pensar e avaliar os eventos da vida e os comportamentos eliciadores de estresse (Sadir \& Lipp, 2009). Quando tais situações estão relacionadas ao contexto laboral, verifica-se a vulnerabilidade ao estresse no trabalho.

Níveis elevados de estresse ocupacional em profissionais da área da saúde, decorrente da discrepância entre as exigências do trabalho e os recursos disponíveis para manejá-las, foram descritos em estudos (R. M. B. Silva \& Moreira, 2019; Barros et al., 2016; Milagres \& Lodi, 2016), especialmente nos profissionais atuantes em nível hospitalar (Guido et al., 2009), em virtude da configuração do trabalho nesse ambiente, caracterizado pela assistência direta a pacientes críticos e/ou em cuidados paliativos, contato com o adoecimento e sofrimento do paciente, contato iminente com a morte, entre outros estressores. As atividades dos profissionais de saúde tendem a ser marcadas por forte tensão, devido às extensas jornadas de trabalho, ao número restrito de trabalhadores e ao desgaste psicoemocional a que estão expostos. Ademais, a necessidade de funcionamento diuturno, que implica a existência de regime de turnos e plantões, tolera a ocorrência de mais de um emprego, situação corriqueira na área da saúde, em que os profissionais mantêm mais de um vínculo empregatício, visando à complementação da renda familiar (Nascimento, Araújo, \& Almeida, 2018).

O processo de trabalho em saúde é um fenômeno complexo e dinâmico, influenciado por mudanças socioeconômicas, políticas e tecnológicas, as quais, por sua vez, modificam as condições laborais e as exigências do contexto de trabalho, potencializando os riscos ocupacionais e psicossociais (Ribeiro, Marziale, Martins, Galdino, \& Ribeiro, 2018). O hospital comparece como um espaço laboral insalubre, penoso e perigoso para os seus trabalhadores, com potencial para vivências de precarização. Caracteriza-se por ser um local propício ao adoecimento físico, aos riscos de acidentes e ao sofrimento psíquico de seus profissionais, ocorrências em ascensão diante das transformações sucedidas no mundo do trabalho nos últimos anos, uma vez que a intensificação laboral, como traço típico da atual fase do capitalismo, trouxe consigo a insegurança gerada pelo medo do desemprego, que faz com que as pessoas sucumbam a regimes e contratos de trabalho precários, percebendo baixos salários em condições de trabalho, geralmente, indignas (Nascimento et al., 2018). Este cenário contemporâneo tem sido considerado, por muitos pesquisadores, uma grande ameaça, 
tornando os trabalhadores de saúde vulneráveis ao estresse ocupacional e ao adoecimento (Ribeiro et al., 2018).

Embora os estudos sobre estresse ocupacional tendam a investigar, em sua grande maioria, os seus efeitos nocivos, outra parte dos estudos tende a investigar as formas com que as pessoas reagem quando estão sob estresse, ou seja, buscam analisar a relação entre o surgimento do estresse e as estratégias de enfrentamento (Lazarus \& Folkman, 1984; Pinheiro, Tróccoli, \& Paz, 2002; Moraes et al., 2016). Lazarus e Folkman desenvolveram um modelo teórico para o entendimento da relação entre estresse e coping. Neste modelo, os eventos estressantes são interpretados como interações entre o indivíduo e o meio, nas quais o impacto ou não dos eventos estressantes é mediado pela avaliação cognitiva que a pessoa faz do evento, bem como dos recursos sociais, psicológicos e culturais disponíveis, ou seja, de suas estratégias de enfrentamento, no momento de ocorrência do evento (Folkman \& Lazarus, 1980; Lazarus \& Folkman, 1984). Dessa forma, de acordo com o modelo apresentado, o coping é considerado um processo adaptativo, ativado quando a relação pessoa-ambiente é vista como estressante, sendo a avaliação cognitiva a razão para as diferenças individuais e grupais na vulnerabilidade ao estresse, dado o seu caráter pessoal (Ramos, Enumo, \& Paula, 2015).

Atualmente, a definição mais aceita para as estratégias de enfrentamento é a utilizada nas pesquisas desenvolvidas por Lazarus e Folkman (1984), os quais definem coping como uma variável individual, representada por um conjunto de esforços empreendidos pelos indivíduos diante de situações que são avaliadas com potencial capacidade ameaçadora de sua integridade e bem-estar, dependentes de fatores pessoais, de exigências situacionais e de recursos disponíveis.

De acordo com Latack (1986), o qual desenvolveu um modelo de compreensão do coping no ambiente de trabalho, dando origem à Escala Coping Ocupacional (ECO), validada para a realidade brasileira por Pinheiro, Tróccoli e Tamayo (2003), a relação entre estresse e coping é entendida como um processo dinâmico, constituída por quatro componentes essenciais, quais sejam: 1) estressores ambientais; 2) avaliação cognitiva desses estressores; 3 ) nível de estresse experienciado pelo indivíduo, seja no plano fisiológico, seja no comportamental; e 4) a utilização de comportamentos ou estratégias de coping. A proposta de Latack em voltar suas pesquisas para o ambiente laboral se justifica pela especificidade deste ambiente e, sobretudo, por perceber que estratégias de enfrentamento consideradas efetivas no ambiente doméstico e contexto interpessoal não têm se mostrado eficazes para lidar com o estresse advindo do ambiente ocupacional.

Pesquisas relacionaram o estresse ocupacional às estratégias de enfrentamento de profissionais de saúde no campo hospitalar, a exemplo de Umann (2011), que, ao investigar 129 enfermeiros atuantes na assistência a pacientes críticos de um hospital universitário, verificou que 33,3\% dos enfermeiros apresentaram estresse elevado e $87,6 \%$ utilizavam estratégias de coping relacionadas ao controle. O estudo de Maturana e Valle (2014) apontou a presença de estresse em $51,6 \%$ dos profissionais de saúde, sendo a comunicação e o relacionamento entre as equipes o fator que mais contribuiu para o estresse. Quanto às estratégias de enfrentamento, foi verificado que os enfermeiros utilizavam, com maior frequência, a resolução de problemas e reavaliação positiva; já os psicólogos e assistentes sociais utilizavam, 
preponderantemente, a resolução de problemas e suporte social. Outras pesquisas (G. A. V. Silva et al., 2017; Teixeira, Gherardi-Donato, Pereira, Cardoso, \& Reisdorfer, 2016; Pereira et al., 2016; N. A. R. Santos, Santos, Silva, \& Passos, 2017) ressaltam que, embora seja identificada a susceptibilidade a que estão submetidos os profissionais de saúde, as estratégias de enfrentamento são capazes de diminuir os níveis de estresse ocupacional, quando utilizadas de modo adequado.

A realidade de demandas e prestação de serviços assistenciais dos hospitais-escola coaduna com a de outros hospitais que não possuem vocação pedagógica ou papel precípuo na formação de mão de obra especializada em saúde, contudo esta última particularidade introduz aspectos diferenciadores, próprios das rotinas dos hospitais-escola, quais sejam: o fato de comportarem programas de formação e atuação profissional, a exemplo das Residências Multiprofissionais em Saúde (RMS), de maneira que os trabalhadores de saúde desenvolvam competências por meio do ensino em prática, visando à articulação entre ensino, pesquisa, extensão e assistência (R. M. B. Silva \& Moreira, 2019). Associada a este elemento, está a existência de diversificados atores protagonizando papéis laborais e sociais neste espaço de atenção à saúde, como docentes, discentes de graduação e de pós-graduação, e profissionais do corpo de saúde, o que colabora para a complexidade das interações socioprofissionais e da coordenação e sincronização dos esforços de trabalho e desempenhos dirigidos ao paciente.

Assim sendo, é válido destacar dois aspectos distintivos da presente pesquisa, realizada em um hospital-escola. O primeiro, em relação ao estudo com residentes multiprofissionais na área da saúde, cujas publicações científicas são consideradas escassas, em virtude, entre outros motivos, da natureza recente da criação das RMS, datada de pouco mais de uma década, para a produção de evidências acerca do tema. O trabalhador residente está sujeito a se deparar com eventos desafiadores, associados a pressões internas e externas, o que pode resultar em situações geradoras de estresse. Tais situações podem oscilar desde sobrecarga de trabalho, alta carga horária, cobranças de diversas naturezas, baixa remuneração por hora trabalhada, instabilidade de vínculo profissional até o não reconhecimento do trabalho desenvolvido no cotidiano dos serviços (R. M. B. Silva \& Moreira, 2019). O segundo, que diz respeito à análise do regime de trabalho de dois grupos profissionais - residentes e empregados públicos - atuantes nesse hospital, em que pese o vínculo empregatício ser um fator promissor a ser investigado, por estar relacionado ao sentimento de segurança, à precarização das relações laborais, entre outros fatores (Nascimento et al., 2018).

Isto posto, o objetivo geral do presente estudo foi comparar a vulnerabilidade ao estresse no trabalho e às estratégias de enfrentamento utilizadas por profissionais de saúde do Hospital Universitário da Universidade Federal de Sergipe (HU-SE). Especificamente, objetivou-se: a) identificar a existência e intensidade da vulnerabilidade ao estresse no trabalho em profissionais de saúde; b) identificar as estratégias de enfrentamento utilizadas pelos participantes; c) verificar a relação entre dados sociodemográficos, ocupacionais, vulnerabilidade ao estresse e estratégias de enfrentamento utilizadas pelos participantes; e d) comparar os resultados referentes à vulnerabilidade ao estresse no trabalho e às estratégias de enfrentamento entre os grupos estudados (residentes e empregados públicos).

A sistematização de conhecimentos científicos sobre o comparecimento da vulnerabilidade ao estresse e de estratégias de enfrentamento em profissionais de saúde, sobretudo aqueles 
detentores de vínculos de trabalho diferenciados e que atuam em contextos hospitalares universitários, pode apontar caminhos de intervenção de finalidade primária ou terciária, no que tange ao estresse laboral, e consubstanciar a produção acadêmica sobre o tema.

\section{Método}

\section{Participantes}

Trata-se de um estudo de caráter quantitativo-descritivo e comparativo, com delineamento transversal. A pesquisa foi realizada com profissionais de saúde empregados públicos, vinculados à Empresa Brasileira de Serviços Hospitalares (EBSERH), e residentes multiprofissionais do Programa Saúde do Adulto e Idoso (SAI), obedecidos os critérios de inclusão e exclusão, além da aceitação desses em participar da pesquisa, mediante assinatura do Termo de Consentimento Livre e Esclarecido.

As Residências em Saúde devem ter duração mínima de dois anos e carga horária de 60 horas semanais, destinadas às profissões da saúde, em regime de dedicação exclusiva (exceto a Medicina) e supervisão docente-assistencial. Atualmente, as profissões a que se destinam são Biomedicina, Ciências Biológicas, Educação Física, Enfermagem, Farmácia, Fisioterapia, Fonoaudiologia, Medicina Veterinária, Nutrição, Odontologia, Psicologia, Serviço Social e Terapia Ocupacional (R. M. B. Silva \& Moreira, 2019).

Na presente pesquisa, foram convidados a participar 40 membros de cada grupo (residentes e empregados públicos), totalizando amostra de 80 profissionais. O tamanho da amostra foi determinado pela composição do Programa de Residência Saúde do Adulto e Idoso (SAI), o qual perfazia 40 estudantes de pós-graduação, no total. Tendo em vista a intenção de reunir um grupo de tamanho compatível para fazer a comparação, o montante da categoria de funcionários da EBSERH também foi de 40 pessoas. Nesta pesquisa, foram considerados profissionais de saúde aqueles que detinham nível superior de escolaridade nas seguintes áreas de formação: Farmácia, Enfermagem, Fonoaudiologia, Fisioterapia, Nutrição, Psicologia, Serviço Social, Odontologia. A exigência quanto às formações mencionadas se deveu ao fato de unicamente estas comporem o Programa de Residência SAI no hospital universitário em questão.

Cabe mencionar que no HU-SE só existem dois programas de residência multiprofissional, SAl e Vigilância Epidemiológica. O programa SAI foi eleito para a pesquisa por atender à exigência de prestação direta de assistência ao paciente. Por não existir a categoria médica no programa, os médicos foram excluídos da amostra, a fim de ser preservada a equivalência de comparação intergrupos (residentes $x$ empregados públicos).

A análise do regime de trabalho dos dois grupos profissionais - residentes e empregados públicos - atuantes nesse hospital revela componentes estressores semelhantes em ambos, tais como: assistência direta ao paciente, contato com o adoecimento e sofrimento do paciente, entre outros (Grazziano, 2009; Guido et al., 2009; A. F. Santos \& Cardoso, 2010), assim como é possível perceber disparidades quanto ao regime de trabalho, no que se refere à carga horária e forma de cumpri-la, vínculo empregatício, acúmulo de funções, entre outras especificidades. Todos estes componentes são fatores que podem contribuir para a vulnerabilidade ao estresse no contexto ocupacional (Paschoal \& Tamayo, 2004) e possíveis diferenciações intergrupo. Por essas razões, considerou-se pertinente o estudo comparativo desses dois grupos. 
A pesquisa contou com os seguintes critérios de inclusão: ser profissional da área da saúde, conforme a especificação desta pesquisa; pertencente a um dos dois grupos profissionais acima descritos (residentes ou empregados públicos); e desenvolver atividades voltadas para a assistência direta ao paciente. Quanto a este último critério de inclusão, ele foi selecionado em razão de ser considerado, na literatura, como um fator estressante (Guido et al., 2009; Santos \& Cardoso, 2010). Como critérios de exclusão, elencam-se os seguintes: não ser profissional da área da saúde; durante a coleta de dados, encontrar-se de férias ou licença; e ser profissional da área da saúde que não exerça as atividades voltadas para a assistência direta ao paciente, ou seja, que exerça atividades voltadas ao ensino, à pesquisa e à administração.

Dos participantes que trabalham nos variados setores do HU-SE, 67 profissionais são do sexo feminino $(83,8 \%)$ e 13 do sexo masculino (16,2\%), entre 22 e 57 anos $(M=30,65$; $\mathrm{DP}=6,71$ ). Além disso, $70 \%$ dos profissionais são praticantes de alguma religião. Quanto à escolaridade, $53,8 \%$ dos profissionais possuem somente ensino superior completo, e os demais apresentam, adicionalmente ao ensino superior, algum nível de pós-graduação (32,5\%: especialização; 12,5\%: mestrado; e 1,2\%: doutorado). Quanto aos aspectos laborais, 57,5\% afirmaram ter recebido treinamento para atuar no setor/unidade de lotação; $72,5 \%$ destes profissionais não possuem outro emprego; $62,5 \%$ trabalham nos turnos da manhã e da tarde; e 51,2\% recebem entre 1 e 3 salários. Ademais, $60 \%$ dos profissionais participantes da pesquisa se ausentaram do trabalho, nos últimos 6 meses, devido a problemas de saúde.

\section{Instrumentos}

Foram aplicados três instrumentos: um Questionário Sociodemográfico e Ocupacional (sexo, idade, escolaridade, religião, tempo de serviço, treinamento, turno de trabalho, outro emprego, carga horária, salário e ausência no trabalho por problemas de saúde), a Escala de Vulnerabilidade ao Estresse no Trabalho (EVENT) (Sisto et al., 2007) e a Escala Coping Ocupacional (ECO) (Pinheiro, Tróccoli, \& Tamayo, 2003). A escolha da EVENT e da ECO deveu-se ao reconhecimento e à aplicação em alguns trabalhos nacionais, somando-se ao fato de serem instrumentos validados no Brasil.

A EVENT é um teste psicológico, composto por 40 itens $(\alpha=0,91)$, o qual avalia circunstâncias do cotidiano laboral que influenciam na conduta do indivíduo e que podem causar estresse, organizadas em três fatores, a saber: (1) Clima e Funcionamento Organizacional, composto por 16 itens $(3,4,5,6,11,13,14,15,16,18,19,25,26,27,36$ e 40); (2) Pressão no Trabalho, composto por 13 itens (1, 2, 12, 17, 24, 28, 29, 30, 33, 34, 35, 38 e 39); e (3) Infraestrutura e Rotina, composto por 11 itens (7, 8, 9, 10, 20, 21, 22, 23, 31, 32 e 37). 0 fator (1) trata do ambiente físico, da relação estabelecida com a chefia, da adequação do salário à função exercida $(\alpha=0,88)$; o fator (2) refere-se ao acúmulo de funções e trabalhos, à responsabilidade excessiva no trabalho e a obrigações maiores que as dos colegas, além da necessidade de ajudá-los $(\alpha=0,85)$. E, por fim, o fator (3) refere-se a jornadas dobradas, doenças constantes ou acidentes pessoais e equipamento precário $(\alpha=0,77)$. O teste é autoaplicável e o tempo de realização é de, aproximadamente, 5 a 10 minutos. Ele possibilita que o indivíduo assinale o quanto uma situação o incomoda em uma escala de 0 a 2 , em que (0) representa "nunca"; (1) representa "às vezes"; e (2) "frequentemente". Sendo assim, o 
somatório total de um respondente na escala pode variar de um mínimo de 0 a um máximo de 80 pontos. Além disso, por meio da pontuação bruta final, é possível avaliar o nível do indivíduo em vulnerabilidade ao estresse no trabalho, entre cinco níveis possíveis, quais sejam: inferior, médio inferior, médio, médio superior e superior, tanto por fator isolado quanto pela totalidade de vulnerabilidade ao estresse, considerando o grupo ocupacional ao qual o indivíduo pertence, conforme Tabela 1 abaixo.

Cabe mencionar que a Lei n. 4.119 (1962), no Art. 13, dispõe que constitui função privativa do psicólogo a utilização de métodos e técnicas psicológicas com os objetivos de diagnóstico psicológico, orientação e seleção profissional, orientação psicopedagógica e solução de problemas de ajustamento.

Tabela 1

Níveis de Vulnerabilidade ao Estresse para a categoria de profissionais de saúde, segundo a EVENT

\begin{tabular}{|l|c|c|c|c|}
\hline $\begin{array}{l}\text { Níveis de } \\
\text { vulnerabilidade }\end{array}$ & $\begin{array}{l}\text { Fator 1 - Clima e } \\
\text { funcionamento } \\
\text { organizacional }\end{array}$ & $\begin{array}{l}\text { Fator 2 - Pressão } \\
\text { no trabalho }\end{array}$ & $\begin{array}{l}\text { Fator 3 - } \\
\text { Infraestrutura e } \\
\text { rotina }\end{array}$ & $\begin{array}{l}\text { Totalidade de } \\
\text { Vulnerabilidade } \\
\text { ao estresse }\end{array}$ \\
\hline Inferior & Até 6 & Até 8 & Até 3 & Até 22 \\
\hline Médio inferior & $7-10$ & $9-13$ & 4 & $23-29$ \\
\hline Médio & 11 & 14 & 5 & 30 \\
\hline Médio superior & $12-16$ & $15-19$ & $6-7$ & $31-39$ \\
\hline Superior & 17 ou mais & 20 ou mais & 8 ou mais & 40 ou mais \\
\hline
\end{tabular}

Fonte: Sisto et al. (2007).

A ECO foi traduzida da versão de Latack (1986) e adaptada para a realidade brasileira por Pinheiro, Tróccoli e Tamayo (2003). Tem o objetivo de mensurar o coping no ambiente ocupacional. Trata-se de uma escala do tipo Likert, composta por 29 itens relacionados a três fatores, a saber: 11 itens relacionados ao fator Controle (1, 2, 3, 4, 5, 6, 7, 8, 9, 10, 11); 9 itens relacionados ao fator Esquiva $(12,13,14,15,16,17,18,19,20)$; 9 itens relacionados ao fator Manejo de Sintomas (21, 22, 23, 24, 25, 26, 27, 28, 29). O Controle refere-se às ações e reavaliações cognitivas proativas $(\alpha=0,78)$; a Esquiva compreende ações e reavaliações que implicam fuga ou evitação $(\alpha=0,77)$ e o Manejo de Sintomas abrange estratégias que se referem às tentativas popularmente aceitas de lidar com os sintomas do estresse $(\alpha=0,81)$. Os itens a serem respondidos variam de intensidade entre 1 e 5, em que (1) significa "nunca faço isso"; (2) "raramente faço isso"; (3) "às vezes faço isso"; (4) "frequentemente faço isso"; e (5) "sempre faço isso". Os escores de cada fator da ECO são obtidos pela média dos itens que a compõem. Desse modo, o fator que apresentar maior média será considerado o mais frequentemente utilizado pela amostra.

\section{Procedimentos}

A coleta de dados foi realizada pelos autores da pesquisa entre os meses de agosto e novembro de 2017, e transcorreu pelo recurso à amostragem por conveniência, nas dependências do HU-SE, mais especificamente, em uma sala de aula reservada para tal, de 
modo que fossem preservados o sigilo e as condições físicas condizentes à aplicação dos instrumentos. Os participantes dirigiam-se a esta sala após serem abordados em seus postos de trabalho e terem aceito o convite dos autores da pesquisa para participar do estudo. Foi solicitada a assinatura do Termo de Consentimento Livre e Esclarecido e o preenchimento anônimo dos instrumentos de pesquisa, aplicados individual ou coletivamente. O projeto de pesquisa recebeu aprovação pelo Comitê de Ética em Pesquisa com Seres Humanos do HUSE (CAAE n. 71103417.6.0000.5546).

\section{Análise dos Dados}

Os dados oriundos dos instrumentos EVENT e ECO, assim como os do Questionário Sociodemográfico e Ocupacional, foram analisados por intermédio de estatísticas descritivas e inferenciais, por meio do software Statistical Package for the Social Sciences (SPSS) - versão 23, quais sejam: medidas de tendência central, medidas de dispersão, frequências absoluta e relativa, teste $U$ de Mann-Whitney, análise de variância $H$ de Kruskal-Wallis e correlação de Spearman ( $\rho)$. A opção pelo uso de testes não paramétricos se deveu ao fato de o tamanho amostral ser reduzido para efeito de comparação (40 indivíduos por grupo comparado), o que, provavelmente, infringia o atendimento de pressupostos básicos para uso de testes paramétricos (normalidade, linearidade, multicolinearidade e homocedasticidade) (Siegel \& Castellan Jr., 2006). Destarte, o uso de técnicas não probabilísticas de amostragem e de testes de significância não paramétricos reduz o grau de certeza na generalização estatística de resultados (Levin, 1987), colaborando para uma abordagem exploratória ao objeto de estudo, com benefícios descritivos e diagnósticos.

\section{Resultados e Discussão}

A apresentação e discussão dos resultados será realizada obedecendo à seguinte sequência: inicialmente, prosseguir-se-á a identificação da existência e intensidade da vulnerabilidade ao estresse no trabalho entre os participantes, assim como as diferenças entre os grupos profissionais. Na sequência, serão identificadas as estratégias de enfrentamento utilizadas e a comparação entre grupos profissionais, com vistas a identificar diferenças significativas. Por fim, traz-se a influência dos dados sociodemográficos e ocupacionais sobre as duas variáveis do estudo (vulnerabilidade ao estresse no trabalho e coping ocupacional).

Foram solicitadas estatísticas descritivas (médias, desvios-padrão, percentis) para os fatores componentes da EVENT, quais sejam: Clima e Funcionamento Organizacional; Pressão no Trabalho; e Infraestrutura e Rotina. As mesmas análises descritivas foram realizadas para o fator geral, o qual reunia a pontuação total no teste. Os escores médios em cada fator (Figura 1) foram cotejados com as tabelas normativas para interpretação por grupo ocupacional, segundo estudo de normatização do teste psicológico EVENT (Sisto et al., 2007). Significa dizer que, quando do estudo de validação e normatização da EVENT, os participantes daquela pesquisa tiveram sua vulnerabilidade ao estresse normatizada segundo o grupo ocupacional em que fossem classificados, entre seis grupos profissionais reunidos. Cada grupo ocupacional tem escores específicos de vulnerabilidade ao estresse, que os distinguem dos escores apresentados pelos outros grupos profissionais previstos pela normatização do teste.

Sendo assim, entre os grupos ocupacionais preconizados pelo teste psicológico EVENT, 
o grupo ocupacional usado como referência para a consideração dos resultados da presente pesquisa foi o Grupo $3^{2}$, constituído por profissões variadas, de nível superior, entre as quais se encontravam as da área da saúde, a exemplo de dentista, enfermeiro, farmacêutico, médico, nutricionista, psicólogo, entre outros. A tabela normativa deste grupo ocupacional preconiza estratos de vulnerabilidade ao estresse que variam de inferior a superior, passando por médio inferior, médio e médio superior, conforme Tabela 1 já apresentada. No caso do fenômeno em tela, vulnerabilidade ao estresse, destacam-se os resultados que estejam classificados nos níveis médio superior e superior, por sinalizarem para quadros acentuados de vulnerabilidade.

Na Figura 1, encontram-se as médias e os desvios-padrão da amostra em cada um dos fatores constitutivos do teste, assim como o resultado do fator geral. A pontuação total média, obtida na EVENT pelos profissionais avaliados, foi de 32,36 ( $D P=10,4)$. Em relação aos fatores que compõem a escala, Clima e Funcionamento Organizacional ( $M=14,37, D P=5,42$ ) foi o que apresentou maior pontuação, a qual está compreendida no estrato médio superior de vulnerabilidade ao estresse, segundo normas de referência do teste sistematizadas na Tabela 1. Pressão no Trabalho ( $M=12,60, D P=5,18)$ posicionou-se no estrato médio inferior e Infraestrutura e Rotina $(M=5,38, D P=2,68)$ posicionou-se no estrato médio de vulnerabilidade.

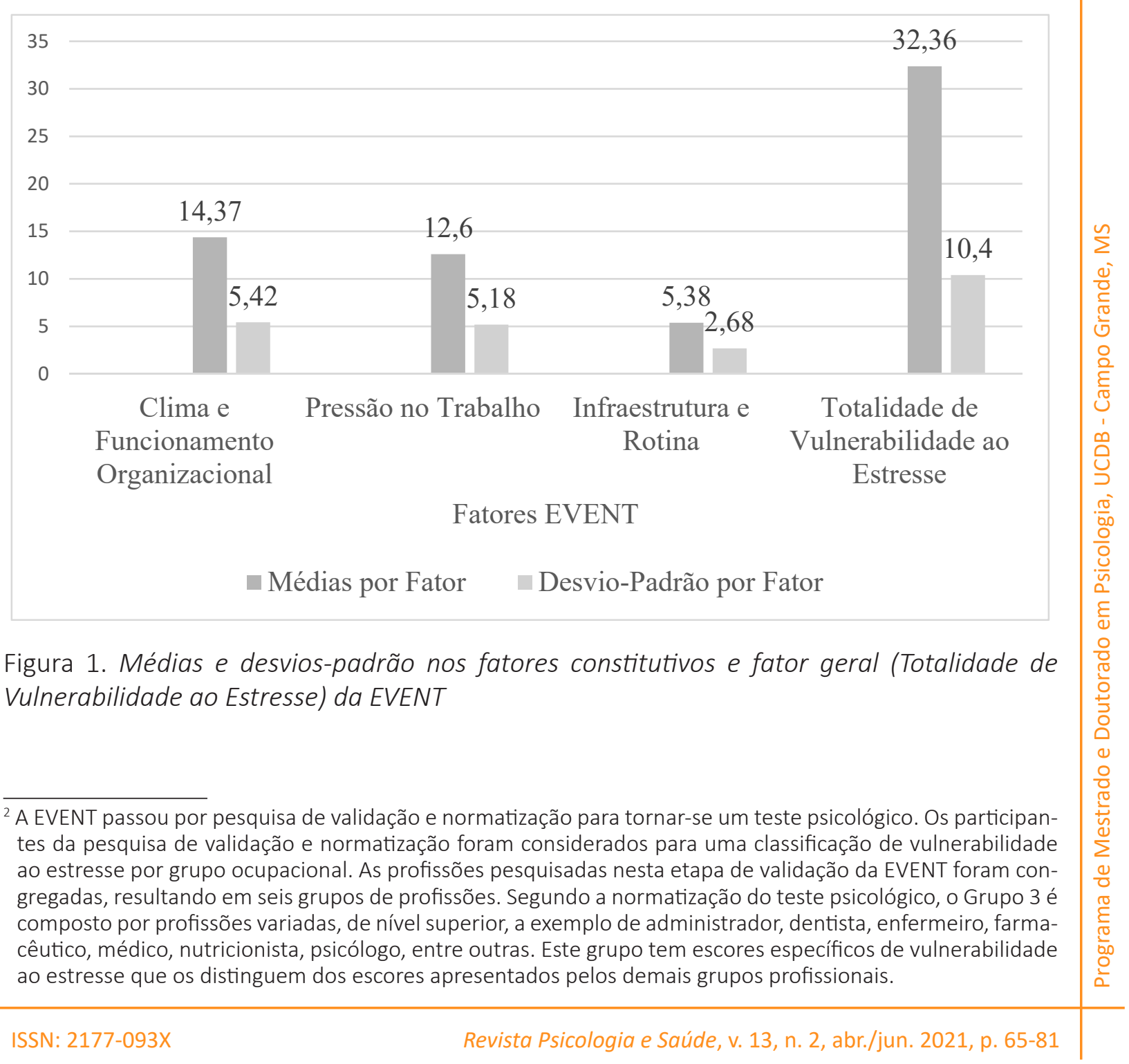


Assim, como pode ser apreendido pelo escore no fator geral, a vulnerabilidade desta amostra ao estresse no trabalho é classificada no estrato médio superior. Esses resultados sugerem que os profissionais que atuam nos diversos setores do HU-SE apresentam alta vulnerabilidade ao estresse no trabalho, ainda que os aspectos componentes do fator Pressão no Trabalho - quais sejam, acúmulo de funções e trabalho, realização de trabalhos que não se referem à função, realizar o trabalho de outro, possuir muita responsabilidade no trabalho diário, necessitar ajudar o colega, ter novas obrigações, prazos para realizar trabalhos, responsabilidade excessiva, ritmo acelerado de trabalho, ter mais obrigações do que os demais colegas e ter de atender muitas pessoas de uma só vez -, aparentemente, não sejam percebidos pelos participantes como situações ameaçadoras, uma vez que foi a menor classificação de vulnerabilidade (estrato médio inferior).

Elevadas frequências de estresse, semelhantes às desta pesquisa, têm sido identificadas em profissionais de saúde, tanto nacionalmente (Maturana \& Valle, 2014) quanto internacionalmente (Tsai \& Liu, 2012). Nesse último estudo, o qual objetivava investigar fatores e sintomas associados ao estresse no trabalho em 775 profissionais de saúde de um hospital, foram encontrados sintomas de estresse relacionado ao trabalho em $64,4 \%$ dos entrevistados. O alto nível de susceptibilidade ao estresse a que os profissionais da saúde estão submetidos em seu ambiente de trabalho está relacionado ao fato de que estes profissionais vivenciam, de modo direto e ininterrupto, o processo de dor, a morte, o sofrimento, o desespero, a angústia, a irritabilidade e demais sentimentos e reações que são desencadeados pelo processo de adoecimento do paciente (Santos \& Cardoso, 2010).

Os achados da presente pesquisa, considerando os escores isolados dos fatores da EVENT, são correspondentes com os do estudo realizado por Ottati e Freitas (2013), com 41 profissionais de saúde de um hospital público da cidade de São Paulo. As autoras obtiveram como resultado que os profissionais estudados apresentaram maior vulnerabilidade ao estresse no fator Clima e Funcionamento Organizacional.

Quando comparados os grupos profissionais componentes da amostra (residentes e empregados públicos) quanto aos fatores da EVENT, não foi encontrada diferença estatisticamente significativa entre os grupos, contrariamente à pesquisa conduzida por Cahú, Santos, Pereira, Vieira e Gomes (2014), que objetivou avaliar o estresse e a qualidade de vida em residentes multiprofissionais e descobriu que um número elevado de residentes (62,2\%) apresentou indicativo de alto estresse. Guido, Silva, Goulart, Bolzan e Lopes (2012) acreditam que os altos níveis de estresse dos residentes multiprofissionais ocorrem devido ao fato de que esses profissionais estão inseridos em um contexto peculiar: expostos a situações estressantes decorrentes tanto do processo de trabalho quanto de ensino-aprendizagem, o que, por sua vez, aumenta a suscetibilidade desses profissionais ao estresse. No presente trabalho, as análises comparativas entre residentes e empregados públicos sugeriram a possibilidade de uma diferença promissora quanto ao fator Clima e Funcionamento Organizacional (escore $U$ de Mann-Whitney $=595,00, z=-1,65, p=0,09$ ), permanecendo a necessidade de aumentar o tamanho da amostra para alcançar resultados estatisticamente significativos.

$\mathrm{Na}$ avaliação do coping, a partir da ECO, as pontuações são direcionadas a estratégias de enfrentamento específicas, quais sejam, controle, esquiva e manejo de sintomas, por meio das médias dos itens que compõem cada estratégia, sem que haja avaliação conjunta e somatória dos valores obtidos, com vistas a gerar uma média total. Portanto, para este 
instrumento, não há escore geral. Assim, o fator que apresentar maior média será considerado o prevalente para os profissionais de saúde estudados.

Na Figura 2, são mostradas as médias obtidas em cada fator componente da ECO. Verificase que a estratégia mais utilizada pelos profissionais de saúde é o controle $(M=3,64 ; D P=$ $0,44)$, apresentando média superior ao ponto médio da escala $(3,0)$, seguindo-se o manejo de sintomas $(M=2,53 ; D P=0,71)$ e a esquiva $(M=2,52 ; D P=0,58)$, praticamente empatados.

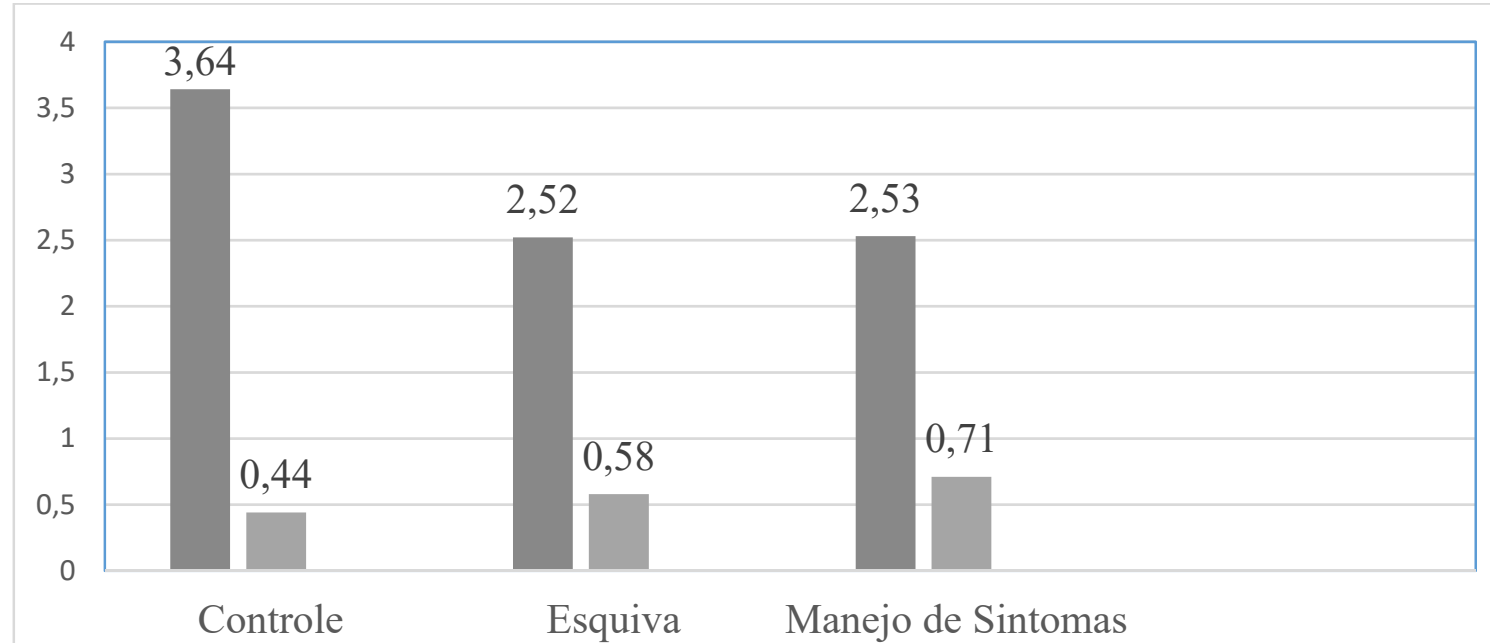

Fatores da ECO

- Médias por Fator $\quad$ Desvio-Padrão por Fator

Figura 2. Médias e desvios-padrão nos fatores constitutivos da ECO

Percebe-se que os profissionais de saúde, diante de situações estressoras, utilizam com mais frequência a estratégia de enfrentamento do tipo controle, que corresponde às ações e às reavaliações cognitivas relacionadas ao enfrentamento, à resolução do problema, uma vez que a média de todos os itens que compõem o fator Controle ficou entre 3 (Às vezes faço isso) e 4 (Frequentemente faço isso). Por outro lado, utilizam com menor frequência a estratégia de esquiva diante de situações estressoras, que corresponde às ações e reavaliações cognitivas de conteúdo escapista, dado que a média de todos os itens que compõem este fator encontra-se entre 2 (Raramente faço isso) e 3 (Às vezes faço isso). Os resultados obtidos assemelham-se aos encontrados em outros estudos (Umann, 2011; Guido et al., 2009), nos quais a estratégia de enfrentamento do tipo controle é mais utilizada, em contrapartida à de esquiva, menos frequente.

Há estudos que evidenciam que o uso da estratégia de enfrentamento esquiva está fortemente relacionado a ambientes altamente estressores, nos quais os indivíduos encontram-se em estado de exaustão emocional, sensação de superexigência e sentimentos de esgotamento e cansaço como consequência do trabalho (Tamayo \& Tróccoli, 2002). Tais constatações justificam a utilização reduzida da estratégia esquiva na população deste estudo, uma vez que, embora a avaliação da vulnerabilidade ao estresse, por meio da EVENT, tenha apontado alta vulnerabilidade, esta não vem caracterizar a população como em estado de exaustão 
emocional. No que tange à comparação dos subgrupos residentes e empregados públicos quanto aos fatores do coping, não foram encontradas diferenças significativas, indicando que não existem estratégias de enfrentamento preferencialmente utilizadas por cada grupo.

O teste do relacionamento entre as variáveis sociodemográficas e ocupacionais e as variáveis psicológicas (vulnerabilidade ao estresse no trabalho e coping ocupacional), empreendido via correlação de Spearman, acusou somente uma relação significativa, qual seja: idade associou-se negativamente com Esquiva ( $\rho=-0,21 ; p=0,05)$. Logo, profissionais mais jovens usam mais recorrentemente a fuga ou evitação de situações estressoras como estratégia de enfrentamento. Estudo conduzido por Contreras, Juarés e Murrain (2008), com trabalhadores de saúde, apresentou resultados congruentes aos encontrados na presente pesquisa, ao demostrar que alguns fatores sociodemográficos e ocupacionais são preditores significativos para o aumento na utilização de todas as estratégias de enfrentamento. Entre estes preditores, encontra-se ter menor idade.

Foram realizadas outras análises inferenciais, dessa vez comparando fatores da ECO com dados sociodemográficos e dados ocupacionais, por meio do teste $U$ de Mann-Whitney. Nesse sentido, foi encontrado que os participantes que não praticam religião (mediana = 2,88 ) utilizam mais que aqueles que praticam alguma religião (mediana $=2,33$ ) a estratégia de enfrentamento do tipo manejo de sintomas ( $U=474,50 ; z=-2,07 ; p=0,03)$. Inversamente, foi encontrado que aqueles que praticam alguma religião (mediana $=3,72$ ) utilizam mais que aqueles que não praticam nenhuma religião (mediana $=3,45$ ) a estratégia de enfrentamento do tipo controle $(U=482,00 ; z=-2,00 ; p=0,04)$.

O resultado da presente pesquisa assemelha-se ao do estudo conduzido por Pargament (1997), ao contrariar o estereótipo de que os indivíduos que utilizam a religião como estratégia de enfrentamento são defensivos, passivos e focados na emoção. O autor demonstra que a associação entre religião e o enfrentamento ao estresse cobre toda uma série de comportamentos, cognições e reavaliações ativas, o que pode explicar a relação entre religião e controle encontrada nesta pesquisa.

Quanto à comparação dos dados ocupacionais com os fatores da ECO, observou-se que aqueles que receberam treinamento (mediana $=2,61$ ) utilizam mais que aqueles que não receberam (mediana $=2,33$ ) a estratégia de enfrentamento do tipo manejo de sintomas ( $U$ = 593,50; Z =- 1,83; $p=0,06)$. De acordo com Umann (2011), a realização de treinamentos pode influenciar na escolha da estratégia de enfrentamento utilizada, na medida que proporciona subsídios para a tomada de decisão e solução de problemas. Contudo os resultados aqui apresentados não corresponderam aos encontrados pela autora mencionada, ao ter avaliado 129 enfermeiros de um hospital universitário. No estudo desta autora, foi encontrado que os enfermeiros que não treinaram para atuar na unidade de lotação utilizavam com maior frequência a estratégia manejo de sintomas, atitudes passivas e permissivas para mediar o impacto do estresse.

Wu, Chi, Chen, Wang e Jin (2010) corroboram tal situação ao afirmarem que a realização de treinamentos proporciona aos profissionais de saúde conhecimentos e habilidades para lidarem com as demandas do trabalho, auxiliando-os no gerenciamento do estresse no ambiente laboral. Por consequência, estes profissionais não precisariam recorrer ao manejo dos sintomas, posto que a ocorrência de sintomas advindos do estresse seria menor.

A não correspondência dos achados da presente pesquisa com os de Umann (2011) e 
de Wu et al. (2010) pode ser justificada pela brevidade do treinamento fornecido, pela não continuidade do treinamento na ocasião de mudança de setor/unidade de lotação, pela inexistência de profissionais preceptores para algumas profissões componentes da residência. No que se refere especificamente à preceptoria, os residentes do estudo de R. M. B. Silva e Moreira (2019) relatam que, muitas vezes, o preceptor não apresenta o preparo necessário para atuar no programa de residência, o que pode dificultar o processo de ensino-aprendizagem e estimular a emergência de relações conflituosas entre preceptor e residente. Assim, a insuficiência de treinamento e de conhecimento dos profissionais, como também a falta de familiarização com as normas e rotinas de cada setor, situações estas associadas a falhas e erros, poderiam contribuir para a utilização de estratégias de enfrentamento mais focadas na administração dos sintomas do estresse do que na solução de problemas (Wu et al., 2010). Percebe-se que, mais que um treinamento fornecido aos profissionais, é preciso que a capacitação seja pautada na lógica da educação permanente e de qualidade.

\section{Considerações Finais}

O ambiente hospitalar e a área da saúde são marcados pela presença de fatores contributivos ao surgimento do estresse ocupacional nos profissionais de saúde. O controle supervisionado, o excesso de trabalho, o lidar diretamente com a vida e a morte, entre outros fatores, alertam para a necessidade de um olhar mais cuidadoso para a saúde mental desses profissionais, dado o caráter deletério que o estresse ocupacional ocasiona à saúde.

Dessa forma, verificar a vulnerabilidade ao estresse no trabalho e as estratégias de enfrentamento utilizadas por profissionais de saúde torna-se essencial para que sejam desenvolvidas medidas de prevenção e promoção da saúde ocupacional, a fim de proporcionar ambiente mais salubre, produtivo e minimizando ao máximo os efeitos prejudiciais do estresse ocupacional. Adicionalmente, comparar os resultados referentes à vulnerabilidade ao estresse no trabalho e às estratégias de enfrentamento entre os grupos estudados (residentes e empregados públicos), bem como identificar a influência de dados sociodemográficos e ocupacionais sobre a variáveis de interesse, presta-se a produzir informações que mapeiem a ocorrência destes fenômenos em contexto de trabalho hospitalar.

Quanto à vulnerabilidade ao estresse, a amostra enquadra-se na posição média superior, com o escore total de 32,36 pontos. Especificamente, entre os fatores que compõem a EVENT, Clima e Funcionamento Organizacional foi o que apresentou maior pontuação (estrato médio superior), seguido pelos fatores Infraestrutura e Rotina (estrato médio) e Pressão no Trabalho (estrato médio inferior). Quando comparados os grupos componentes da amostra (residentes e empregados públicos) quanto aos fatores da EVENT, não foram encontrados dados significativos, contrariamente ao que se esperava, em função de a literatura apontar que as Residências Multiprofissionais em Saúde apresentam um contexto peculiar, em função dos processos de trabalho e de ensino-aprendizagem, que podem ser avaliados como estressores (R. M. B. Silva \& Moreira, 2019).

Quanto às estratégias de enfrentamento, verificou-se que a estratégia mais utilizada pelos profissionais de saúde é o Controle, e que o Manejo de sintomas e a Esquiva são menos utilizadas. Na comparação dos grupos profissionais, não foram encontradas diferenças sig- 
nificativas, indicando que não existem estratégias de enfrentamento preferencialmente utilizadas por cada grupo.

Constata-se, neste estudo, que os profissionais de saúde investigados, empregados públicos e residentes do Hospital Universitário de Sergipe, encontram-se com vulnerabilidade ao estresse preocupante, mesmo utilizando com maior frequência a estratégia de enfrentamento Controle, a qual pressupõe ações e reavaliações cognitivas proativas na resolução do estresse.

Os achados desta pesquisa ficaram limitados na representatividade da variedade de profissionais que habitam o contexto hospitalar investigado, posto que não estão incluídos o grupo profissional de estatutários e a categoria médica, e na abordagem não probabilística de amostragem, a qual fragiliza a generalização destes resultados. Outro limite comparece na ausência de investigação do efeito de variáveis, como tempo de atuação profissional e de formação sobre os fenômenos de interesse neste estudo.

A não inclusão do grupo de profissionais estatutários se deu pelo número reduzido de profissionais pertencentes às categorias profissionais estudadas e que atuam diretamente na assistência aos pacientes. Ainda assim, a atenção dada ao estudo de profissionais de vínculos empregatícios diferentes, em especial a residentes, é algo a ser reconhecido neste trabalho, uma vez que tal aspecto rareia nas publicações. Os residentes multiprofissionais não são profissionais com vínculos efetivos junto aos serviços de saúde. Os problemas encontrados no contexto da prática influenciam diretamente o processo de formação, a exemplo da quantidade insuficiente de preceptoria de campo, do número exacerbado de horas trabalhadas durante os dois anos de duração da residência e da utilização do residente em substituição a categorias profissionais insuficientes no serviço (Silva \& Moreira, 2019), sinalizando para vivências de precarização do trabalho. Novos estudos merecem ser conduzidos, a fim de aprofundar o conhecimento sobre a realidade laboral de profissionais da saúde e fenômenos atrelados, em especial, com profissionais residentes, dada a escassez de publicações com esta população, aspecto que o presente estudo colabora para minorar.

Sugere-se, ainda, a realização de estudos com interesse em avaliar a (co)relação entre estresse ocupacional e outros aspectos, tais quais: suporte social, ansiedade, exaustão emocional, suporte organizacional, índices de absenteísmo, entre outros. Além disso, estudos em setores específicos, tanto da rede pública quanto da rede privada de saúde, tais como UTI, Pediatria, entre outros, carecem de ser realizados, dadas as particularidades destes ambientes. Igualmente, a heterogeneidade profissional da amostra investigada aponta para áreas com perfis de atuação diferentes, que poderiam ser alvo de estressores ou fazerem uso de estratégias de enfrentamento específicos, suspeita que merece investigação mais pormenorizada.

Os resultados deste estudo contribuem para o avanço do conhecimento multiprofissional em saúde do trabalhador, fornecendo subsídios à área da saúde em geral, para o desenvolvimento de medidas preventivas e terapêuticas ao estresse ocupacional e para a promoção e proteção da saúde e bem-estar dos profissionais que lidam diretamente com a assistência a pacientes hospitalizados. Quando de sua comprovada ocorrência, devemos estar atentos aos efeitos do estresse nos profissionais de saúde, como também atentar para os impactos deste nos serviços de saúde oferecidos à população. 


\section{Referências}

Barros, M. M., Almeida, S. P., Barreto, A. L. P., Faro, S. R. S., Araújo, M. R., \& Faro, A. (2016). Síndrome de Burnout em médicos intensivistas: estudos em UTIs de Sergipe. Temas em Psicologia, 24(1), 377-389. doi:http://dx.doi.org/10.9788/TP2016.1-26

Cahú, R. A., Santos, A. C., Pereira, R., Vieira, C., \& Gomes, S. (2014). Estresse e qualidade de vida em residência multiprofissional em saúde. Revista Brasileira de Terapias Cognitivas, 10(2), 76-83. doi:http://dx.doi.org/110.5935/1808-5687.20140013

Contreras, F., Juarés, F., \& Murrain, E. (2008). Influencia del Burnout la calidad de vida y los factores socioeconómicos en las estrategias de afrontamiento. Pensamiento Psicológico, 4(11), 29-44.

Folkman, S., \& Lazarus, R. (1980). An analysis of coping in a middle-age community sample. Journal of health and social behavior, 21(1), 219-239.

Grazziano, E. S. (2009). Estratégia para redução do estresse e Burnout entre enfermeiros hospitalares. (Tese de doutorado, Escola de Enfermagem da Universidade de São Paulo Universidade de São Paulo, São Paulo). Recuperado de http://www.teses.usp.br/teses/ disponiveis/7/7139/tde-14052009-101907/pt-br.php

Guido, L., Silva, R., Goulart, C., Bolzan, M., \& Lopes, L. (2012). Síndrome de Burnout em residentes multiprofissionais de uma universidade pública. Revista Escola de enfermagem da USP, 46(6), 1477-1483. doi: http://dx.doi.org/10.1590/S0080-62342012000600027

Guido, L., Umann, J., Stekel, L., Linch, G., Silva, R., \& Lopes, L. (2009). Estresse, coping e estado de saúde de enfermeiros de clínica médica em um hospital universitário. Ciência Cuidado e Saúde, 8(4), 615-621. doi: http://dx.doi.org/10.4025/cienccuidsaud.v8i4.9690

Latack, J. C. (1986). Coping with job stress: measures and future directions for scale development. Journal of Applied Psychology, 71(3), 377-385.

Lazarus, S., \& Folkman, S. (1984). Stress, appraisal, and coping. New York: Springer.

Lei n. 4.119, de 27 de agosto de 1962 (1962). Dispõe sobre os cursos de formação em psicologia e regulamenta a profissão do psicólogo. Recuperado em http://www.planalto. gov.br/ccivil_03/LEIS/1950-1969/L4119.htm

Levin, J. (1987). Estatística aplicada a ciências humanas (2a ed.). São Paulo: Harbra Ltda.

Mansur, A. P., \& Favarato, D. (2011). Mortality due to cardiovascular diseases in Brazil and in the metropolitan region of Sao Paulo: a 2011 update. Arquivos Brasileiros de Cardiologia, 99(2), 755-761.

Maturana, A. P., \& Valle, T. G. (2014). Estratégias de enfrentamento e situações estressoras de profissionais no ambiente hospitalar. Psicologia Hospitalar, 12(2), 2-23.

Milagres, C. S., \& Lodi, J. C. (2016). Formas de enfrentamento e saúde do enfermeiro diante de estressores ocupacionais. Pensar Acadêmico, 14(2), 100-107.

Moraes, F., Benetti, E. R. R., Herr, G. E. G., Stube, M., Stumm, E. M. F., \& Guido, L. A (2016). Estratégias de coping utilizadas por trabalhadores de enfermagem em terapia intensiva neonatal. Revista Mineira de Enfermagem, 20, 1-8. doi:http://dx.doi. org/10.5935/1415-2762.20160036

Nascimento, T. C. C., Araújo, M. R. M., \& Almeida, S. P. (2018). Precarização do emprego em um hospital público de Sergipe: um estudo de caso com profissionais da enfermagem. Revista de Ciências da Administração, 20 (Edição Especial), 117-129. doi:http://dx.doi. org/10.5007/2175-8077.2018 V20nespp117 
Ottati, F., \& Freitas, V. (2013). Avaliação da qualidade de vida e vulnerabilidade ao estresse no contexto hospitalar. Estudos Interdisciplinares em Psicologia, 4(1), 15-29. doi:http:// dx.doi.org 10.1590/S1413-82712011000100007

Pargament, K. (1997). The psychology of religion and coping: theory, research, practice. New York: Guilford Press.

Paschoal, T., \& Tamayo, A. (2004). Validação da Escala de Estresse no Trabalho. Estudos de Psicologia, 9(1), 45-52.

Pereira, S. S., Teixeira, C. A. B., Reisdorfer, E., Vieira, M. V., Gherardi-Donato, C. S., \& Cardoso, L. (2016). A relação entre estressores ocupacionais e estratégias de enfrentamento em profissionais de nível técnico de enfermagem. Texto Contexto Enfermagem, 26(4), 1-8.

Pinheiro, F. A., Tróccoli, B., \& Paz, M. G. T. (2002). Aspectos psicossociais dos distúrbios osteomusculares (Dort/LER) relacionados ao trabalho. In A. M. Mendes, L. O. Borges, \& M. C. Ferreira (Eds.), Trabalho em transição, saúde em risco (pp. 65-85). Brasília: Universidade de Brasília.

Pinheiro, F. A., Tróccoli, B., \& Tamayo, M. (2003). Mensuração de coping no ambiente ocupacional. Psicologia: Teoria e Pesquisa, 19(2), 153-158. doi:http://dx.doi. org/10.1590/0102-37722003000200007

Ramos, F. P., Enumo, S. R. F., \& Paula, K. M. P. (2015). Teoria motivacional do coping: uma proposta desenvolvimentista de análise do enfrentamento do estresse. Estudos da psicologia, 32(2), 269-279. doi:http://dx.doi.org/10.1590/0103-166X2015000200011

Ribeiro, R. P., Marziale, M. H. P., Martins, J. T., Galdino, M. J. Q., \& Ribeiro, P. H. V. (2018). Estresse ocupacional entre trabalhadores de saúde de um hospital universitário. Revista Gaúcha de Enfermagem, 39(1), 1-6. doi:http://dx.doi.org/10.1590/1983-1447.2018.65127

Sadir, M. A., \& Lipp, N. E. M. (2009). As fontes de estresse no trabalho. Revista de Psicologia da IMED, 1(1), 114-126.

Santos, A. F. (2010). Determinantes psicossociais da capacidade adaptativa: um modelo teórico para o estresse. (Tese de Doutorado, Universidade Federal da Bahia, Salvador, BA). Recuperado de https://ri.ufs.br/handle/riufs/1898

Santos, A. F., \& Cardoso, C. L. (2010). Profissionais de saúde mental: estresse, enfrentamento e qualidade de vida. Psicologia: Teoria e Pesquisa, 26(3), 543-548. doi:http://dx.doi. org/10.1590/S0102-37722010000300017

Santos, N. A. R., Santos, J., Silva, V. R., \& Passos, J. P. (2017). Estresse ocupacional na assistência de cuidados paliativos em oncologia. Cogitare Enfermagem, 22(4), e50686. doi:http:// dx.doi.org/10.5380/ce.v22i4.50686

Siegel, S., \& Castellan Jr., N. J. (2006). Estatística não-paramétrica para ciências do comportamento (2a ed.). Porto Alegre: Artmed.

Silva, G. A. V., Silva, G. S. A., Silva, R. M., Andolhe, R., Padilha, K. G., \& Costa, A. L. S. (2017). Estresse e coping entre profissionais de enfermagem de unidades de terapia intensiva e semi-intensiva. Revista de Enfermagem Universidade Federal de Pernambuco, 11(2), 922931. doi:http://dx.doi.org/10.5205/reuol.10263-91568-1-RV.1102sup201707

Silva, R. M. B., \& Moreira, S. N. T. (2019). Estresse e residência multiprofissional em saúde: compreendendo significados no processo de formação. Revista Brasileira de Educação Médica,43(4),157-166.doi:http://dx.doi.org/10.1590/1981-52712015v43n4RB20190031 
Sisto, F. F., Baptista, M. N., Noronha, A. P. P., \& Santos, A. A. A. (2007). Escala de Vulnerabilidade ao Estresse no Trabalho - EVENT. São Paulo: Vetor.

Stults-Kolehmainen, M. (2013). The interplay between stress and physical activity in the prevention and treatment of cardiovascular disease. Frontiersand Physiology, 4(346), 1-4. doi:http://dx.doi.org/10.3389/fphys.2013.00346

Tamayo, M. R., \& Tróccoli, B. (2002). Exaustão emocional: relações com a percepção de suporte organizacional e com as estratégias de coping no trabalho. Estudos de Psicologia, 7(1), 37-46. doi:http://dx.doi.org/10.1590/S1413-294X2002000100005

Teixeira, C. A. B., Gherardi-Donato, E. C. S., Pereira, S. S., Cardoso, L., Reisdorfer, E. (2016). Estresse ocupacional e estratégias de enfrentamento entre profissionais de enfermagem em ambiente hospitalar. Enfermería Global, 44(1), 299-309.

Tsai, Y. C., \& Liu, C. H. (2012). Factors and symptoms associated with work stress and healthpromoting lifestyles among hospital staff: a pilot study in Taiwan. BWC Health Service Research, 12(199). doi:http://dx.doi.org/10.1186/1472-6963-12-199

Umann, J. (2011). Absenteísmo na equipe de enfermagem no contexto hospitalar. Ciência, Cuidado e Saúde, 10(1), 184-190. doi:http://dx.doi.org/10.4025/cienccuidsaude. v10i1.11867

Wu, H., Chi, T., Chen, L., Wang, L., \& Jin, L. (2010). Occupational stress among hospital nurses: cross-sectional survey. Journal of Advanced Nursing, 66(3), 627-634. doi:http://dx.doi. org/10.1111/j.1365-2648.2009.05203x

Recebido em: 22/09/2019

Última revisão: 07/02/2020

Aceite final: 01/04/2020

\section{Sobre os autores:}

Rafael Lima de Matos: Especialista em Saúde do Adulto e Idoso pela Universidade Federal de Sergipe (UFS). Graduado em Psicologia pela UFS. Atualmente é psicólogo organizacional e do trabalho no Hospital Universitário de Lagarto (HUL-UFS). E-mail: rafaelmattos123@hotmail.com, Orcid: http://orcid.org/0000-0002-9460-7043

Marley Rosana Melo de Araújo: Pós-doutorado em Psicologia Social pela Universidade Federal da Paraíba (UFPB). Doutorado em Psicologia pela Universidade Federal do Pará (UFPA). Graduada em Psicologia pela UFPA. Atualmente é docente do departamento de Psicologia na Universidade Federal de Sergipe (UFS). E-mail: marleymeloaraujo@gmail.com, Orcid: http://orcid.org/0000-0002-5161-6863 
